\title{
Kinematics of the wire-driven parallel robot MARIONET using linear actuators
}

\author{
J-P. Merlet
}

\begin{abstract}
Wire-driven parallel robots are special types of Gough-Stewart platform in which the rigid extensible legs are substituted by extensible wires. Usually wire length change is obtained by coiling the wire on a drum actuated by a rotary motor. We present here a robot using linear actuator and a pulley system allowing a higher modularity of the actuation system. The kinematics of this redundant system (the system has 7 wires), taking into account the elasticity of the wires, is presented. Inverse kinematics may be solved either by first choosing the wire tensions, the control vector being then a linear function of the tensions or by solving directly a system of non linear equations whose unknowns are the components of the control vector. Forward kinematics is a much more complex issue, involving the solving of a large system. We present a solving approach but this problem remains an open issue.
\end{abstract}

\section{INTRODUCTION}

\section{A. THE MARIONET ROBOT}

Wire-driven parallel robots are special types of GoughStewart platform in which the rigid extensible legs are substituted by extensible wires. Although known for some time [1], [11], [12], [14], [20], [21] they are currently extensively studied [2], [3], [5], [7], [9], [17], [18] as they offer a larger workspace and high end-effector velocities. Usually wire length change is obtained by coiling the wire on a drum actuated by a rotary motor (see for example the FALCON [11] or SEGESTA [9] robots). If some accuracy is required this solution has the drawback that a special guide must be manufactured on the drum to establish an accurate linear relationship between the measured rotation of the drum and the length of the coiled wire. As the dimension of the drum is limited (to reduce the inertia and for allowing the coiling) the amount of coiled wire is also limited and consequently the modularity of the system is reduced.

We have started designing a new robot, called Marionet, with modularity as focus point: as the robot performances are highly dependent upon the robot geometry (in a broad sense) we wish to be able to quickly modify it in order to adapt the robot to the task at hand. Being able to change the minimal and maximal wire lengths is an important part of this modularity as these parameters play an important role for the robot workspace, accuracy and maximal velocities.

This has motivated us to consider a new coiling system based on a linear actuator and a pulley system (figure 1). The linear actuator (Copley Motion type M 2506) has a platform that slides along a rod using a single rail stage. The motion of the platform is measured by a linear incremental encoder with an accuracy of $1 \mu \mathrm{m}$.

J-P. Merlet is with INRIA, BP 93, 06902 Sophia-Antipolis, France Jean-Pierre.Merletesophia.inria.fr

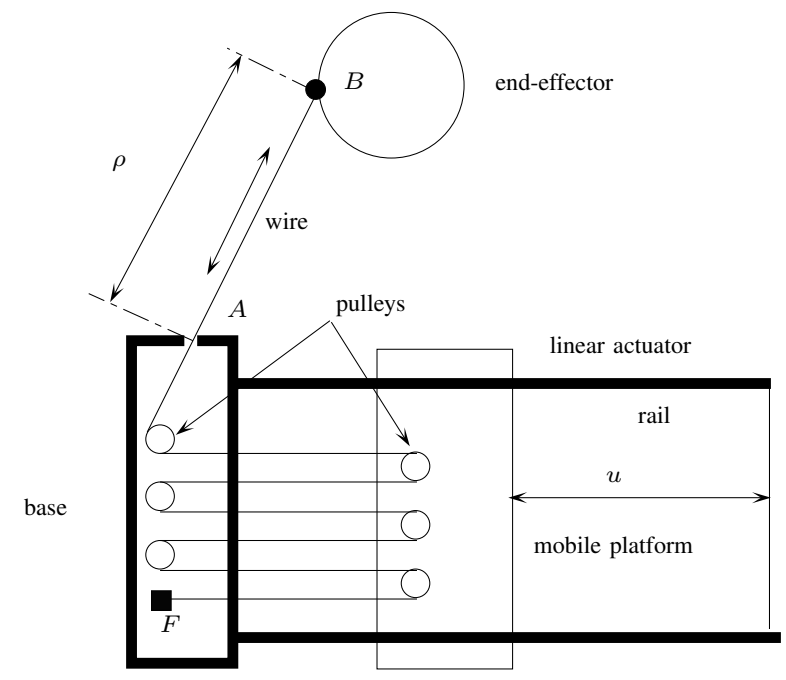

Fig. 1. The wire system used to actuate the Marionet robot

One extremity of the wire is attached to a fixed point $F$ on the base and from this point the wire goes alternatively to pulleys that are fixed either on the mobile platform or on the base. The wire goes from a final pulley on the base through a fixed opening, whose location is $A$, to the robot end-effector at point $B$ : the distance between $A$ and $B$ will be denoted $\rho$ and the points $A, B$ are called the anchor points of the robot. Hence the motion of the linear actuator is amplified by the pulleys system by a factor $K$, which is an even number. Note that a direct drive may be obtained if an extremity of the wire is connected to the mobile platform and goes directly to the final pulley,.

Seven such wire systems are fixed on standard profile elements that constitutes the frame of the robot (figure 2).

The robot is controlled by a PC under Linux including electronic boards that are connected to power amplifiers. Potential applications that will be considered for this robot are:

- rehabilitation: we will use the robot to measure the motion of human joints and establish various models according to the patient. Then the precise force feedback that may be obtained from wire-driven robot may be used to implement a rehabilitation protocol or for sport training [15], [16]

- service robotics: in this domain we intend to explore the use of Marionet as a windows washing machine or as an assistant device for elderly people [10]

- entertainment robotics: we intend to explore the use of 


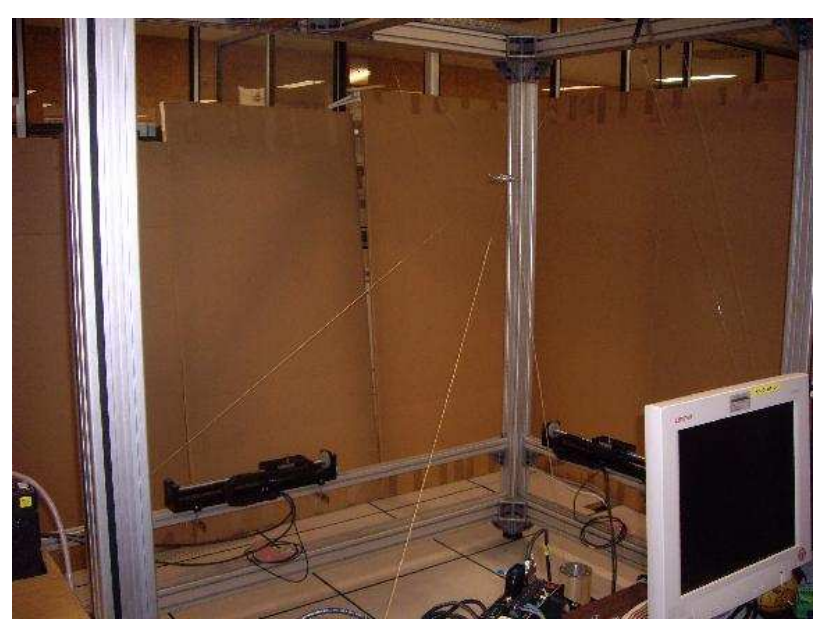

Fig. 2. The Marionet robot

the robot for movie maker (allowing new angle of view for cameras and special effects based on out of habit actors motion) and fast scenic changes in opera and theaters

- rescue robotics: we intend to use the robot as an independent, portable crane for assisting rescuer during major natural catastrophe [19]

- industrial robotics: Marionet will allow extremely fast motion of the end-effector that may be convenient for pick-and-place operations

In all these applications modularity is extremely important and Marionet combines both a modular mechanical system and a design software that will allow to determine what is the best geometry of the robot for the task at hand.

Still the development of a new robot has to go through the study of basic problems and we address in this paper the kinematics issues. Apart of the original mechanical structure of the wire system our contribution will be to take into account the wire elasticity in the inverse and forward kinematics, an issue that, to the best of our knowledge, has not been addressed for wire-driven parallel robots.

\section{KINEMATICS}

Currently the wires of the robot are standard fishing wires with high elasticity. This elasticity may be modeled with Hooke's law: if $L_{0}$ is the length of the unloaded wire and $L$ its length under tension, then the tension $\tau$ in the wire may be calculated as

$$
\tau=k \frac{L-L_{0}}{L}
$$

where $k$ characterizes the material of the wire. The total length of the wire may be written as

$$
L=\rho+V+K u
$$

where $u$ is the actuator position with respect to a given point on the actuator axis and $V$ is a fixed constant that is the length of the wire between $A$ and $F$. We may assume that $L_{0}, V, K$ are known constants. Clearly the wire deformation must be taken into account for the kinematics of the robot as soon as its elasticity is high. If we assume a small velocity of the end-effector the tension $\tau$ in the wires are related to the wrench $\mathcal{F}$ exerted on the platform by

$$
\mathcal{F}=\mathbf{H} \tau
$$

where $\mathbf{H}$ is usually called the inverse jacobian matrix of the robot, which is pose dependent. The elements of $\mathbf{H}$ are known analytical functions of the pose parameters. We define a reference frame $\mathcal{R}=(O, \mathbf{x}, \mathbf{y}, \mathbf{z})$ and uses the coordinates in $\mathcal{R}$ of a point $C$ on the platform to parameterize the position of the end-effector. A mobile frame $C, \mathbf{x}_{\mathbf{m}}, \mathbf{y}_{\mathbf{m}}, \mathbf{z}_{\mathbf{m}}$ is attached to the platform and the Euler angles are used to represent the orientation of the platform. If $\mathbf{n}_{\mathbf{i}}$ is the unit vector giving the direction of wire $i$, then the $i$-th column $H_{i}$ of $\mathbf{H}$ may be written as

$$
H_{i}=\left(\begin{array}{ll}
\left(\mathbf{n}_{\mathbf{i}}\right. & \mathbf{C B}_{\mathbf{i}} \times \mathbf{n}_{\mathbf{i}}
\end{array}\right)
$$

\section{A. INVERSE KINEMATICS}

\section{1) INVERSE KINEMATICS ALGORITHMS:}

For the inverse kinematics the pose of the end-effector is known and we will consider one wire to determine what should be its control $u$. First the length $\rho$ may be determined: indeed the pose of the end-effector allows one to determine the coordinates of $B$ in the reference frame, while the coordinates of $A$ in the same frame are known. Hence the components of the vector $\mathbf{A B}$ can be determined and $\rho$ is the norm of this vector.

If the end-effector is submitted to known forces/torques (e.g. the gravity) it is possible to solve in $\tau$ the linear system (3) using methods that are classical for wire-driven robot [4], [5], [9] ensuring that all elements of $\tau$ are positive to avoid having slack wires. The control $u$ is then calculated using $(1,2)$ as

$$
u=\frac{k L_{0}}{K(k-\tau)}-\frac{\rho+V}{K}
$$

The drawback of this approach is that both $u$ and $\tau$ have limits (the linear actuator has a limited stroke and the $\tau$ must be positive and also lower than a fixed threshold to avoid breaking the wire). With this method we may ensure that the limits on $\tau$ are verified but cannot guarantee that the limits on $u$ are also satisfied.

We may note that the wire tension may be expressed as

$$
\tau=\frac{k\left(\rho+V-L_{0}+K u\right)}{\rho+V+K u}
$$

The constraints $\tau<\tau_{\max }$ may thus be written as $K u(k-$ $\left.\tau_{\max }\right)<\tau_{\max }(\rho+V)-k\left(\rho+V-L_{0}\right)$. Usually $k$ will much larger than $\tau_{\max }$ and hence $k-\tau_{\max }$ will be positive. Consequently we may determine an upper bound for $u$ as $\left(\tau_{\max }(\rho+V)-k\left(\rho+V-L_{0}\right)\right) /\left(k-\tau_{\max }\right)$. The same approach used for the constraint $\tau>\tau_{\min }$ allows one to determine a lower bound for $u$.

Using equations $(1,2)$ equations (3) is now a non-linear system of 6 equations in the seven unknowns $u$, from which 
we must determine the solutions that are included between the lower and upper bounds that have been determined in the previous paragraph.

Inverse kinematics may be used in two cases:

- to move the robot from its current pose to another one, that may be far from the current one. In that case the control vector $u$ may be quite different from the current one

- during a robot motion in which case we are looking for solutions that are close to the current values of $u$. Here computation should be real time

The number of methods for solving a non-linear system are limited and we are using interval analysis with the library ALIAS that is developed in our laboratory. This approach allows one to determine solutions of a system which are included in a pre-defined domain and its computation time decreases with the size of the domain. These properties make the method appropriate for addressing the two above issues.

Different strategies may be used to solve the system (3):

- Algorithm 1: we consider (3) as an under-constrained system of 6 equations in the $7 u_{i}$. The ranges for the $u_{i}$ will describe the actuator full stroke if no information on the final $u_{i}$ is available and we will stop the algorithm as soon as a solution is found. For a real time use the range for the $u_{i}$ will be a small interval centered around the last known value

- Algorithm 2: we fix the value of one of the $u_{i}$ and consider (3) as a square system of equations. The value of the fixed $u_{j}$ may be determined by using the new value of $\rho_{j}$ and equation (5) with a fixed value for $\tau_{j}$ within the range $\left[\tau_{\min }, \tau \max \right]$. Up to now we have been able to determine if this system may have multiple solutions.

\section{2) NUMERICAL EXAMPLES:}

We are considering two possible designs of MARIONET with the anchor point coordinates presented in table I. The second design which differs only by the coordinates of the $B$ points (indicated in parenthesis) and is called T-platform (also called 3-2-1 [8]). In this design there are only 3 different anchor points: one hosting three wire extremities and two attached each with 2 wires. More precisely wires $(1,4,5)$ are connected at the same $B_{1}$ points, $(2,6)$ at the point $B_{2}$ and $(3,7)$ at $B_{3}$.

We have identified the value of $k$ by applying various known loads on a single wire and measuring its length. This has shown that Hooke's law was real close from the wire behavior and have obtained $k=383 N$. The amplification ratio of the actuator was $K=2$ and the mass of the platform is $0.02 \mathrm{~kg}$. The allowed range for $u$ is $[-20,20]$ and is identical for all actuators.

Using Algorithm 1 and the range [-20,20] for all seven $u$ we find a solution in 14 seconds on a Dell D620. If we restrict the range for the $u$ to be centered at the last measured value and fix the width of the range to $1 \mathrm{~mm}$, then a solution is found in 2 seconds. This is unfortunately too large for real time use.
COORDINATES IN CENTIMETER OF THE ANCHOR POINTS $A$ AND $B$ WITH RESPECT TO THE REFERENCE AND MOBILE FRAME

\begin{tabular}{|c|c|c|c|}
\hline & $\mathrm{x}$ & $\mathrm{y}$ & $\mathrm{z}$ \\
\hline$A_{1}$ & 0 & 60 & 0 \\
\hline$A_{2}$ & 180 & 120 & 0 \\
\hline$A_{3}$ & 180 & 0 & 0 \\
\hline$A_{4}$ & 20 & 0 & 180 \\
\hline$A_{5}$ & 20 & 120 & 180 \\
\hline$A_{6}$ & 180 & 120 & 180 \\
\hline$A_{7}$ & 180 & 0 & 180 \\
\hline \hline$B_{1}$ & $-10(-10)$ & $-5(0)$ & 0 \\
\hline$B_{2}$ & $10(10)$ & $-5(10)$ & 0 \\
\hline$B_{3}$ & $0(10)$ & $10(-10)$ & 0 \\
\hline$B_{4}$ & $-5(-10)$ & $-10(0)$ & $10(0)$ \\
\hline$B_{5}$ & $-5(-10)$ & $10(0)$ & $10(0)$ \\
\hline$B_{6}$ & $5(10)$ & 10 & $10(0)$ \\
\hline$B_{7}$ & $5(10)$ & -10 & $10(0)$ \\
\hline
\end{tabular}

For algorithm 2 we assign to $u_{1}$ a value so that the wire tension is the middle of the allowed range for the tension. The square system may be solved using the Newton-Raphson scheme with the drawback that this scheme may not converge (or converge to a solution such that one, or more, wire tension lie outside its allowed range). If the range for all $u$ is $[-20,20]$ the algorithm find a solution in 12 milliseconds. If we restrict the range for the $u$ to be centered at the last measured value and fix the width of the range to $1 \mathrm{~mm}$, then a solution is found in 2 milliseconds which is compatible with a real-time use.

Using a continuation method we may calculate the solution in $u_{j}, j>1$ for a varying $u_{1}$. For example we have considered a planar version of MARIONET with only 4 wires and we have calculated the solutions in $u_{2}, u_{3}, u_{4}$ when $u_{1}$ was varying from 0.1 to $10 \mathrm{~cm}$ (figure 3 ): it may be seen that the solution are roughly linearly dependent upon $u_{1}$. This linear dependency was expected. Indeed the solution in

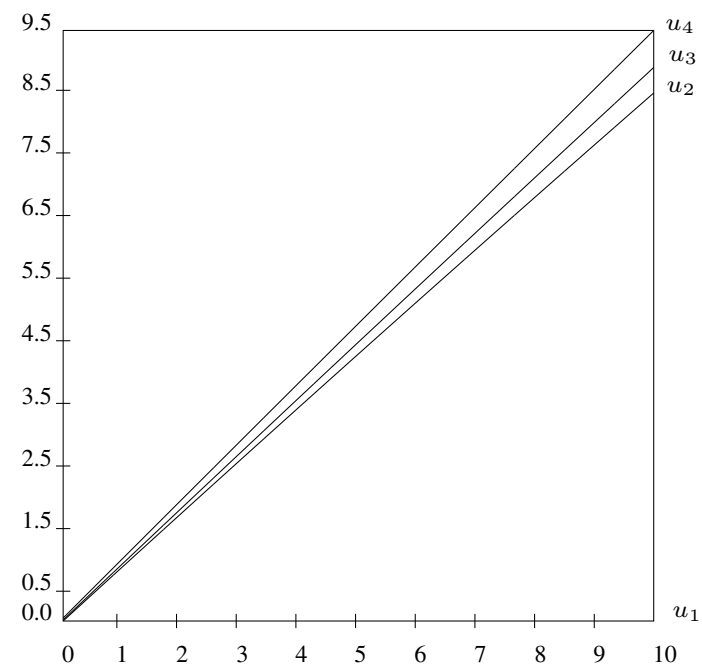

Fig. 3. Solution of the inverse kinematics for the control vector of a planar version of MARIONET when $u_{1}$ is changing

$\tau_{j}, j>1$ of equation (3) will be linear in $\tau_{1}$. Considering 
now equation (6) it may be seen that in the denominator we have $W=\rho+V>>K u$ and consequently we may write

$$
\begin{aligned}
\tau= & \frac{k\left(\rho+V-L_{0}+K u\right)}{W(1+K u / W)} \\
& \approx \frac{k\left(\rho+V-L_{0}+K u\right)(1-K u / W}{W} \\
& \approx \frac{k\left(\rho+V-L_{0}+K u\right)}{W}
\end{aligned}
$$

Although $\rho+V-L_{0}$ is also a function of $u$ it may be assumed that the change in the wire length will be sufficiently small so that this term will be approximately constant and consequently $\tau$ will be a linear function of $u$. Hence $\tau_{j}$ is a linear function of $u_{j}$ but also in $\tau_{1}$ which is linear in $u_{1}$ : consequently $u_{j}$ will be approximately linear in $u_{1}$ as observed. Using this linear approximation we may update the search domain for the $u_{j}$ to find a solution for an arbitrary $u_{1}$.

\section{FORWARD KINEMATICS}

Being given the control vector $u$ the unknowns are the seven $\rho$ and the six pose parameters. We have six equations coming from the force/torque equilibrium (3) and seven equations relating the $\rho$ to the pose parameters (i.e. the inverse kinematic equations of a classical parallel robots). Hence the forward kinematics is obtained as a solution of a square system of 13 non-linear equations. But we may already assume that solving this system will not be an easy task as it amounts to solve at the same time the forward kinematics of a Gough platform (an already difficult task) and in addition the static equilibrium equations with complex elastic elements (a problem which is already difficult in the case where the elastic elements are springs [6]).

Using $(1,2)$ we may calculate $\rho$ as a function of $\tau$ :

$$
\rho=\frac{k L_{0}}{k-\tau}-V-K u
$$

Being given the limits $\left[0, \tau_{\max }\right]$ on $\tau$ we may deduce a range for $\rho$ from this equation. The orientation angle are naturally bounded while the coordinates of $C$ should be such that $C$ lie within the hull of the $A$ anchor points.

\section{A. Planar case}

We may consider an almost planar case in which the planar end-effector is attached to the frame with only four wires and moves roughly within an horizontal plane. The motion of the end-effector will not be exactly planar as the compensation of gravity requires that the wires do not lie in an horizontal plane. Furthermore with 4 wires we can control only 3 pose parameters and therefore cannot compensate the motion of the end-effector along the vertical direction.

Using a minimal parameter set for the pose we have 6 unknowns (the 3 coordinates of $C$ and the rotation angles) and 4 equations for the inverse kinematics that involve the four $\rho$. Mechanical equilibrium provides 6 additional equations and we end up with a system of 10 equations in 10 unknowns.

\section{B. Spatial case}

For the spatial case the system of equations may be transformed into a system of algebraic equations in order to apply classical solving algorithms such as Groëbner basis or the continuation method. However these methods have a complexity which grows exponentially with the number of unknowns and we know that these methods were already at their limits when studying the forward kinematics of classical Gough platform. As we are considering a much more complex problem it appears that these methods may not be appropriate without a difficult in-depth analysis of the system. Another method, that has been successful for the Gough platform [13], is based on interval analysis which requires to restrict the solving problem to determine solution within a bounded domain, which is the case as already mentioned.

However it has been shown that the chosen set of pose parameters was not the most appropriate for using interval analysis [13]. A better set of pose parameters is to use as unknowns the coordinates of 4 of the $B$ points i.e. 12 unknowns, leading to a total of 19 unknowns (the 12 coordinates and the $7 \rho$ ). If we assume that the selected set of $B$ points is $B_{1}, B_{2}, B_{3}, B_{4}$ (assuming that they are not coplanar) we know that we can find a set of 4 scalar constants $l_{1}^{j}, l_{2}^{j}, l_{3}^{j}, l_{4}^{j}$ such that $l_{1}^{j}+l_{2}^{j}+l_{3}^{j}+l_{4}^{j}=1$ and with

$$
\mathbf{O B}_{\mathbf{j}}=l_{1}^{j} \mathbf{O B}_{\mathbf{1}}+l_{2}^{j} \mathbf{O B}_{\mathbf{2}}+l_{3}^{j} \mathbf{O B}_{\mathbf{3}}+l_{4}^{j} \mathbf{O B}_{\mathbf{4}}
$$

Hence the 7 equations inverse kinematic equations are functions only of the 12 coordinates of $B_{1}, B_{2}, B_{3}, B_{4}$ while the 6 equations of (3) are functions of the same unknowns and of the $\rho$. Six additional equations may be obtained by writing that the distances between pairs of points in the set $\left\{B_{1}, B_{2}, B_{3}, B_{4}\right\}$ are known scalars and we end up with a square system of 19 equations in 19 unknowns.

For the T-platform the pose parameters may be reduced to the 9 coordinates of $B_{1}, B_{2}, B_{3}$ and we obtain a reduced system of 16 equations in 16 unknowns. Note that this system may be further simplified by considering the two inverse kinematic equations for two wires sharing the same anchor points $B_{i}$. These equations may written as $\left(x_{i}-x a_{l}\right)^{2}+\left(y_{i}-\right.$ $\left.y a_{l}\right)^{2}+\left(z_{i}-z a_{l}\right)^{2}=\rho_{l}^{2}$ where $x_{i}, y_{i}, z_{i}$ are the coordinates of $B_{i}$ and $x a_{l}, y a_{l}, z a_{l}$ the coordinates of the anchor point on the base. The difference between the two equations is linear in $x_{i}, y_{i}, z_{i}$ and as 4 independent pairs of such equations may be considered we may solve them to determine 4 unknowns, thereby reducing the number of unknowns to 12 . However in the numerical examples we have not considered this reduced system.

Note that equation (8) may also be used in the planar case with a total of 13 unknowns (the 9 coordinates of the $B_{i}$ and the four $\rho$ ) and a total of 13 equations ( 4 from the inverse kinematics, 6 from the mechanical equilibrium and 3 from the distances between $\left.B_{1}, B_{2}, B 3\right)$.

\section{1) NUMERICAL EXAMPLES:}

In a first part of our experiment we have considered the problem of finding all the solutions of the forward kinematic 
problem. It appears quickly that the solving time was heavily sensitive on the bounds given for the $\rho$ and hence on the allowed $\tau_{\max }$. We have considered as possible value for $\tau_{\max }$ either $10 \mathrm{~N}$ or $20 \mathrm{~N}$. Various tests were performed with different values for the $u_{i}$.

\section{Spatial case}

The ranges for the $x_{i}, y_{i}, z_{i}$ were fixed respectively to $[30,160]$, [30,110],[0, 170]. For the T-platform with the bounds for $\tau$ fixed to $[0,10] \mathrm{N}$ we get a single solution for the forward kinematics in about 10 minutes while for $\tau$ in $[0,20]$ two solutions are found in about one hour. These solutions are presented in figure 4,5 . It may be seen that the

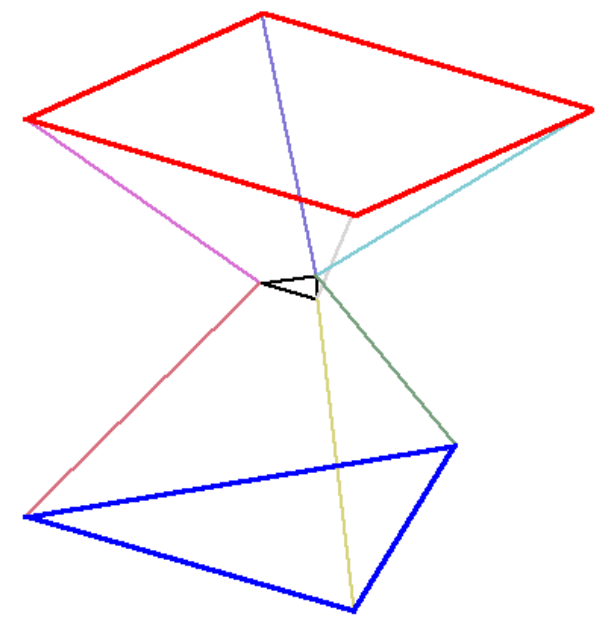

Fig. 4. First solution of the forward kinematics for the T-platform

main transformation between the two solutions is a rotation around the main branch of the "T" although a translation of the anchor point $B_{1}$ with 3 wires is also observed. For the first solution the coordinates of this are $(85.14,54.78$, $100.36)$ and $(93.45,55.65,101.6)$ for the second solution.

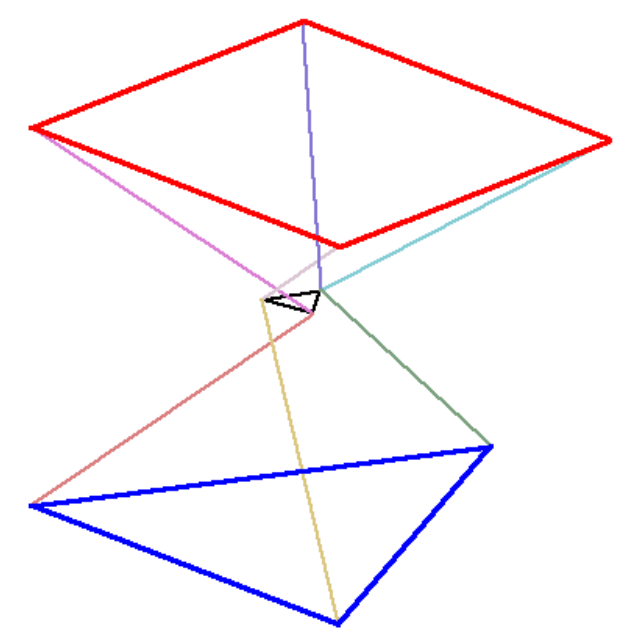

Fig. 5. Second solution of the forward kinematics for the T-platform

For the general platform and $\tau_{\max }=10 N$ we have been able to show that there was a single solution. For $\tau_{\max }=20 \mathrm{~N}$ we have shown that there was at least 2 solutions (presented in figure 6,7 ) but we have been unable to complete the calculation. For real time calculation we

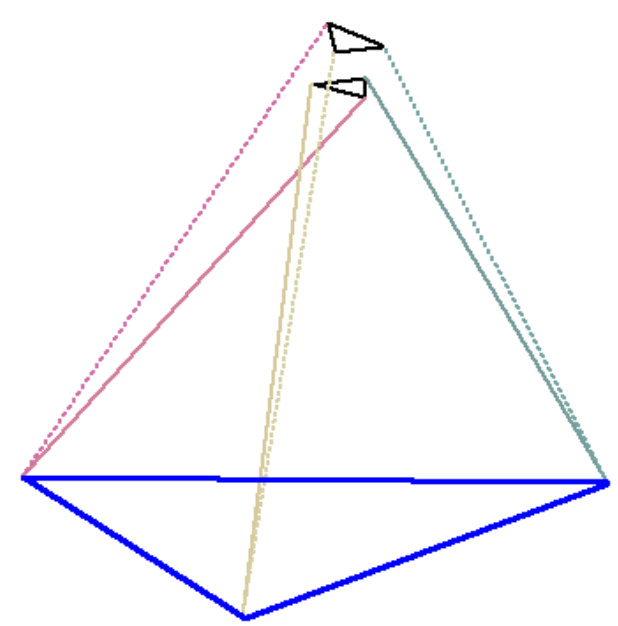

Fig. 6. The location of the 3 lower points of the end-effector

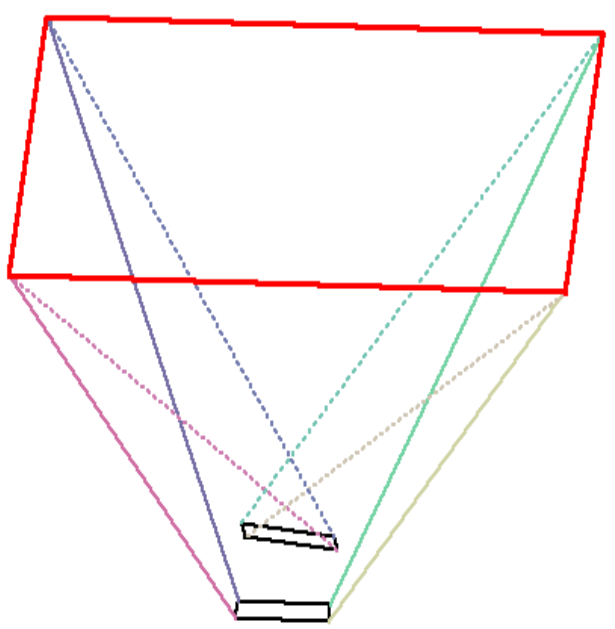

Fig. 7. The location of the 4 upper points of the end-effector

have used the inverse kinematics to determine the control vector for a given pose $P$ and then we have added to each element of this vector a random perturbation in the range $[-1,1] \mathrm{mm}$. Assuming that the location of the $B_{i}$ points were in a range centered at the location of $B_{i}$ for the pose $P$ with a width of $1 \mathrm{~mm}$ we are able to prove that there is a single solution of the forward kinematics in the box in a computation time between 5 and $7 \mathrm{~ms}$. It must be noted that in our trial the Newton scheme was able to converge to the correct solution in less than $1 \mathrm{~ms}$.

\section{Planar case}

The planar case involves solving a system of 13 equations in 13 unknowns if the full pose of the end-effector has to 
be determined. If we use a large value of $\tau_{\max }$ we have found an example with 4 solutions (figure 8) that have been determined in 22 minutes. If we restrict $\tau_{\max }$ to 20 and 10 $\mathrm{N}$ only 2 solutions are valid and are found in a computation of 12 minutes and 7 minutes respectively.

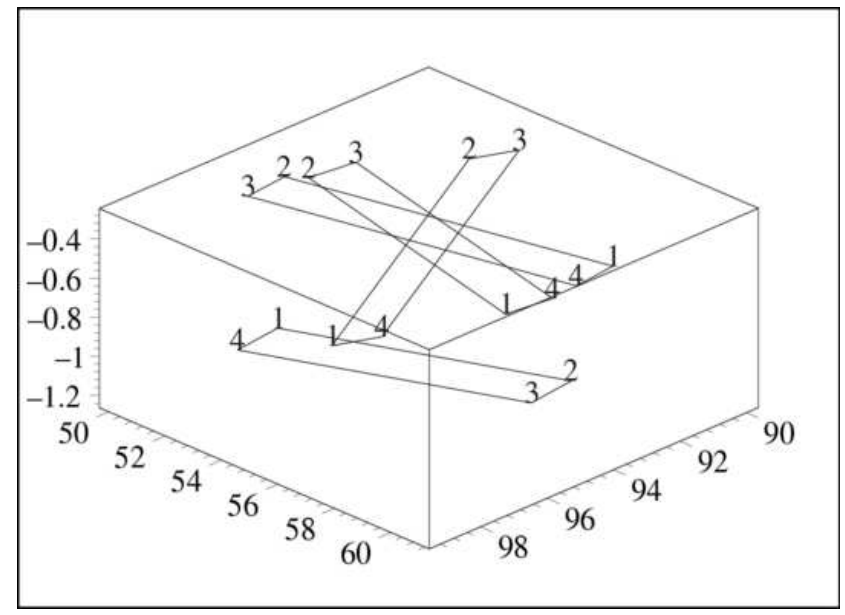

Fig. 8. The four different poses that are solution of a forward kinematics problem for the almost planar robot

The maximal vertical deviation from the manipulator plane is $1.22 \mathrm{~mm}$ while the minimal one is $0.38 \mathrm{~mm}$. These small deviations indicate that it may be interesting to look at the full planar case, assuming no motion along the vertical axis. In that case we get a system in 10 unknowns (the 6 coordinates of the three $B_{i}$ points and the $4 \rho$ ) in 10 equations ( 3 for the distances between the $B_{i}, 4$ for the inverse kinematic and 3 mechanical equilibrium equations). Assuming a large $\tau_{\max } 4$ solutions are found in $1 \mathrm{mn} 20 \mathrm{~s}$ while 4 solutions are also found for $\tau_{\max }=20$ in $29 \mathrm{~s}$ and 2 solutions for $\tau_{\max }=10$ in $3 \mathrm{~s}$.

If we compare the solutions obtained for the $6 \mathrm{D}$ case to the solutions of the planar case in term of mean distances between the four corresponding anchor points of the endeffector we find that the maximal mean distance is $5.3 \mathrm{~cm}$ while the minimal one is $0.16 \mathrm{~cm}$. Hence the planar case may be used to define restricted search spaces for the $6 \mathrm{D}$ case allowing a lower computation time. Our test have shown that by using this strategy the computation time of the $6 \mathrm{D}$ case may be reduced to less than 30 seconds.

\section{CONCLUSION}

We have considered the kinematic issue regarding a new wire-driven parallel robot having an original actuation scheme, taking into account the elasticity of the wire. Compared to parallel robot with rigid links, kinematics is more complex for wire-driven robot. Inverse kinematics is however still manageable, even in real-time. As for all parallel robot the forward kinematics is much more complex. A real time treatment is possible even in the most general case but finding all solutions is computer intensive. It appears that the solutions exhibit some complicated symmetries and a further understanding of these symmetries may allow to reduce the search space, thereby leading to a large reduction in computation time. The maximal number of solutions is also an open issue.

\section{REFERENCES}

[1] Albus J., Bostelman R., and Dagalakis N. The NIST ROBOCRANE. J. of Robotic Systems, 10(5):709-724, July 1993.

[2] Behzadipour S. and Khajepour A. Design of reduced dof parallel cable-based robots. Mechanism and Machine Theory, 39(10):10511065, October 2004.

[3] Bosscher P. and Ebert-Uphoff I. Disturbance robustness measures for underconstrained cable-driven robots. In IEEE Int. Conf. on Robotics and Automation, pages 4206-4212, Orlando, May, 16-18, 2006.

[4] Bosscher P. and Ebert-Uphoff I. Wrench-based analysis of cabledriven robots. In IEEE Int. Conf. on Robotics and Automation, pages 4950-4955, New Orleans, April, 28-30, 2004.

[5] Bruckmann T., Pott A., and Hiller M. Calculating force distributions for redundantly actuated tendon-based Stewart platforms. In ARK, pages 403-412, Ljubljana, June, 26-29, 2006.

[6] Duffy J. Statics and Kinematics with Applications to Robotics. Cambridge University Press, New-York, 1996.

[7] Fattah A. and Agrawal S.K. On the design of cable-suspended planar parallel robots. ASME J. of Mechanical Design, 127(5):1021-1028, September 2005.

[8] Geng Z. and Haynes L.S. A 3-2-1 kinematic configuration of a Stewart platform and its application to six degree of freedom pose measurements. Robotics and Computer-Integrated Manufacturing, 11(1):23-34, March 1994.

[9] Hiller M. and others . Design, analysis and realization of tendon-based parallel manipulators. Mechanism and Machine Theory, 40(4):429445, April 2005.

[10] Homma K. and Arai T. Upper limb motion assist system with parallel mechanisms. In 2nd Japan-France Congress on Mechatronics, pages 388-391, Takamatsu, November, 1-3, 1994.

[11] Kawamura S. and others . High-speed manipulation by using parallel wire-driven robots. Robotica, 18(1):13-21, January 2000.

[12] Landsberger S.E. and Sheridan T.B. A new design for parallel link manipulator. In Proc. Systems, Man and Cybernetics Conf., pages 812-814, Tucson, 1985.

[13] Merlet J-P. Solving the forward kinematics of a Gough-type parallel manipulator with interval analysis. Int. J. of Robotics Research, 23(3):221-236, 2004.

[14] Ming A. and Higuchi T. Study on multiple degree of freedom positioning mechanisms using wires, Part 1, Concept, Design and Control. Int. J. Japan Soc. Prec. Eng., 28(2):131-138, June 1994.

[15] Morizono T., Kurahashi K., and Kawamura S. Realization of a virtual sports training system with parallel wire mechanism. In IEEE Int. Conf. on Robotics and Automation, pages 3025-3030. Albuquerque, April, 21-28, 1997.

[16] Morizono T., Kurahashi K., and Kawamura S. Analysis and control of a force display system driven by parallel wire mechanism. Robotica, 16(5):551-563, September 1998.

[17] Ottaviano E. and Ceccarelli M. Numerical and experimental characterization of singularity of a six-wire parallel architecture. Robotica, 25(3):315-324, May 2007.

[18] Pusey J. and others . Design and workspace analysis of a 6-6 cable-suspended parallel robot. Mechanism and Machine Theory, 139(7):761-778, July 2004.

[19] Tadokoro S. and others . A portable parallel manipulator for search and rescue at large-scale urban earthquakes and an identification algorithm for the installation in unstructured environments. In IEEE Int. Conf. on Intelligent Robots and Systems (IROS), pages 1222-1227, Kyongju, October, 17-21, 1999.

[20] Tadokoro S. and others . On fundamental design of wire configuration of wire driven parallel manipulators with redundancy. In Japan-USA Symp. on Flexible Automation, pages 151-158, Boston, July, 7-10, 1996.

[21] Viscomi B.V., Michalerya W.D., and Lu L-W. Automated construction in the ATLSS integrated building systems. Automation in Construction, 3(1):35-43, May 1994. 